\title{
Ruptured middle colic artery aneurysm
}

\author{
STEPHEN WHITEHEAD \\ M.B., F.R.C.S.
}

Kent and Canterbury Hospital, Canterbury, Kent CT1 3NG

\begin{abstract}
Summary
A case of rupture of an aneurysm of the middle colic artery treated successfully by colectomy is described. The diagnosis and management of mesenteric artery branch aneurysm are discussed.
\end{abstract}

\section{Introduction}

Rupture of an aneurysm of the visceral arteries is a well recognized cause of abdominal pain. The old term, abdominal apoplexy, suitably describes the sudden and catastrophic events that may overtake a patient.

The splenic and renal arteries are the most likely to be responsible, and have been identified as such for many years, but recent reports have included the other visceral arteries and their branches.

\section{Case history}

A 38-year-old woman was admitted to hospital in January 1978 with a 17-hr history of sudden onset of lower abdominal pain, shifting to the epigastrium and accompanied by an episode of non-bloody diarrhoea, following which she collapsed.

She had suffered from systemic lupus erythematosus and hypertension since 1965. In 1977, Libman-Sacks endocarditis of the mitral valve was diagnosed following an episode of gangrene of the right foot due to arterial embolism for which she was anti-coagulated. She was taking warfarin, prednisolone and labetalol at the time of admission.

On examination, she was cold and pale. The pulse was $86 / \mathrm{min}$ and blood pressure was $60 / 40 \mathrm{mmHg}$. A pan-systolic heart murmur was heard, radiating to the apex. Signs of peritonitis were present in the upper abdomen, and there were no masses to feel. Chest and abdominal radiographs showed no abnormality. Her haemoglobin was $10.4 \mathrm{~g} / \mathrm{dl}$ and white cell count $10.6 \times 10^{9} / 1$. A diagnosis of either mesenteric vascular occlusion or a perforated peptic ulcer was made.

Laparotomy was performed through a right paramedian incision. There was free blood in the peritoneal cavity, and a $2.5 \mathrm{~cm}$ ruptured aneurysm of the middle colic artery was found. The site of rupture was partially occluded by blood clot. There had been no bleeding into the mesentery. Two further aneurysms, 1 and $0.5 \mathrm{~cm}$ in diameter, both intact, were found proximal to the rupture. Other visceral arteries were examined and no further aneurysm was found. A limited hemicolectomy with end-toend anastomosis of the ascending colon to the left transverse colon was performed. She made an uneventful recovery. Histology showed a ruptured aneurysm, containing laminated thrombus, with no specific aetiological features and, in particular, none of systemic lupus erythematosus.

\section{Discussion}

Aneurysm of branches of the superior mesenterie artery is rare; Deterling (1971) records only 4 colige artery aneurysms in a series of 1424 visceral artery? aneurysms. A total of 7 have been reported to date (1978) (Buehler, Dailey and Lazarevic, 1976; Stanley, Thompson and Fry, 1970).

Approximately two-thirds of mesenteric artery branch aneurysms are mycotic, with a history of endocarditis, and about a quarter are idiopathic (De Bakey and Cooley, 1953). Although there was a history of endocarditis, there was nothing histologically to suggest that the aneurysm presented here was mycotic. Her age does not favour atherosclerosis and there was no evidence of major arterial disease elsewhere.

The 2 cases of Buehler et al. (1976) both presented with an intra-peritoneal haemorrhage, although bleeding into the mesentery may occur (Deterling, 1971).

If the diagnosis is made early enough and the aneurysm is peripheral, then treatment is reasonably simple, involving resection of an appropriate length of colon. An aneurysm at the root of the superior mesenteric artery, however, is a more formidable proposition (Kostuk and Silver, 1972).

Small aneurysms of the branches of the mesenteric arteries (most of those recorded are $1-2 \mathrm{~cm}$ in diameter) may go unnoticed at laparotomy or postmortem, being hidden in the mesenteric fat, so their 


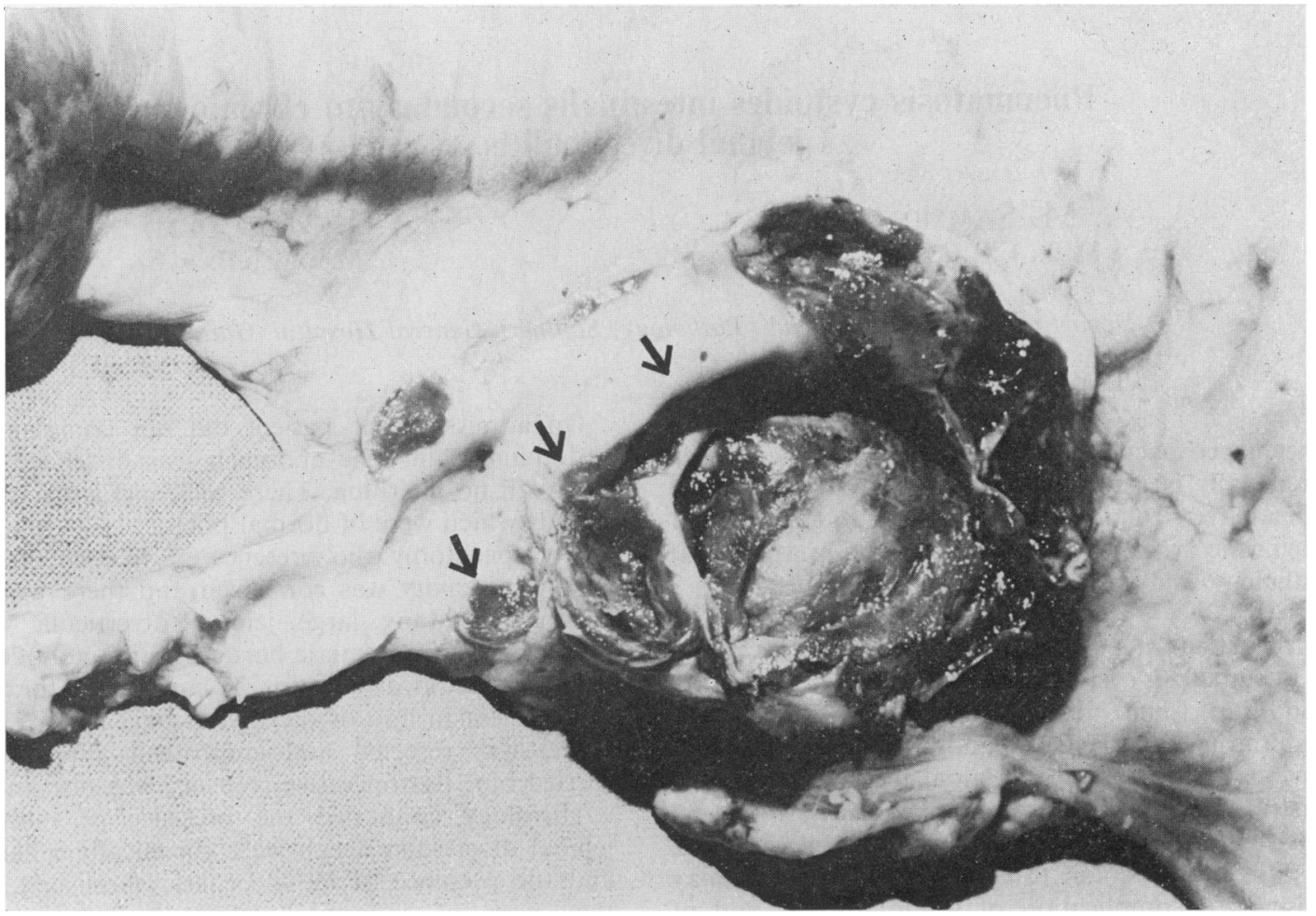

FIG. 1. Resected specimen showing the three aneurysms (arrowed).

true incidence is unknown. Idiopathic retroperitoneal haemorrhage is possibly due to rupture of one of these small aneurysms.

Increasing use of abdominal angiography has brought more visceral aneurysms to light, raising the question of surgical intervention if discovered incidentally. About $50 \%$ of the cases of Deterling (1971) came to surgery. Stanley et al. (1970) advise surgery in all cases once their presence has been discovered.

Only speculation on the fate of an asymptomatic mesenteric artery branch aneurysm is possible until more is known about the natural history of this condition. Minimal signs and symptoms may be present over 2 or 3 days, as slight leakage occurs, before the final and often fatal rupture (Buehler et al., 1976).

In view of the established mortality and morbidity of visceral artery aneurysms (Smith and Hill, 1967), their presence justifies surgical treatment provided operation does not entail an undue risk to the patient.

\section{Acknowledgments}

I thank Dr D. A. Lillicrap and Mr M. R. Williams for permission to report their case.

\section{References}

Buehler, P.K., Dailey, T.H. \& Lazarevic, B. (1976) Spontaneous rupture of colic artery aneurysms. Diseases of the Colon and Rectum, 19, 671.

De BAKeY, M.E. \& CoOleY, D.A. (1953) Successful resection of mycotic aneurysm of superior mesenteric artery: Case report and review of literature. American Surgeon, 19, 202.

Deterling, R.A. (1971) Aneurysm of the visceral arteries. Journal of Cardiovascular Surgery, 12, 309.

KostuK, W.J. \& SilveR, M.D. (1972) Superior mesenteric artery aneurysm. Canadian Journal of Surgery, 15, 86.

Smith, G.W. \& Hill, C.H. (1967) Aneurysms of the branches of the abdominal aorta: Diagnosis, management and results. Surgery, 61, 509.

Stanley, J.C., Thompson, N.W. \& Fry, W.J. (1970) Splanchnic artery aneurysms. Archives of Surgery, 101, 689. 